\title{
THE CONTRIBUTION OF EEG RECORDINGS TO THE AUDIOVISUAL RECOGNITION OF WORDS IN UNIVERSITY STUDENTS WITH DYSLEXIA
}

\author{
Pavlos Christodoulides $^{1}$, Victoria Zakopoulou ${ }^{2}$, Katerina D. Tzimourta $^{3}$, \\ Alexandros T. Tzallas ${ }^{4}$, \& Dimitrios Peschos ${ }^{1}$ \\ ${ }^{I}$ Faculty of Medicine, University of Ioannina, (Greece) \\ ${ }^{2}$ Department of Speech and Language Therapy, University of Ioannina, (Greece) \\ ${ }^{3}$ Department of Electrical and Computer Engineering, University of Western Macedonia, (Greece) \\ ${ }^{4}$ Department of Informatics and Telecommunications, University of Ioannina, (Greece)
}

\begin{abstract}
Dyslexia is one of the most frequent specific learning disorders which has often been associated with deficits in phonological awareness mainly caused by auditory and visual inabilities to recognize and discriminate phonemes and graphemes within words. Neuroimaging techniques like EEG recordings have been widely used to assess hemispheric differences in brain activation between students with dyslexia and their typical counterparts. Although dyslexia is a lifelong disorder which persists into adulthood, very few studies have been carried out targeting in adult population. In this study, we examined the brain activation differences between 14 typical (control group) and 12 university students with dyslexia (experimental group). The participants underwent two tasks consisting of 503 -word groups characterized by different degrees of auditory and visual distinctiveness. The whole procedure was recorded with a 14-sensor sophisticated wearable EEG recording device (Emotiv EPOC+). The findings from the auditory task revealed statistically significant differences among the two sets of groups in the left temporal lobe in $\beta$, $\gamma$ and $\delta$ rhythms, in the left occipital lobe in $\beta$ rhythm, and in the right prefrontal area in $\alpha, \beta$ and $\gamma$ rhythms, respectively. The students with dyslexia reported higher mean scores only in $\delta$ rhythm in the left temporal lobe, and in $\alpha, \beta$ and $\gamma$ rhythms in the right prefrontal area. Concerning the visual task, statistically significant differences were evident in the left temporal lobe in $\beta, \gamma$ rhythms, in the occipital lobe in $\alpha, \beta$ and $\delta$ rhythms, in the parietal lobe in $\beta$ rhythm, and in the right occipital lobe in $\delta, \beta$ and $\gamma$ rhythms. The students with dyslexia reported higher mean scores only in the $\delta$ rhythm of both the left and right occipital lobe. The results indicate that there are differences in the hemispheric brain activation of students with or without dyslexia in various rhythms in both experimental conditions, thus, shedding light in the neurophysiological discrepancies between the two groups. It also lays great emphasis on the necessity of carrying out more studies in adult population with dyslexia.
\end{abstract}

Keywords: Dyslexia, EEG, audiovisual recognition, university students.

\section{Introduction}

Dyslexia is one of the most frequent specific developmental learning disorders, affecting $5-15 \%$ of school-aged children although estimates vary widely depending on the language and culture (American Psychiatric Association, 2013). It has often been related to severe deficits in reading and spelling skills which often co-occur with dysfunctional phonological processing (Snowling, 2000) that persist in adult life (Paulesu et al., 2001), difficulties in phonological representations and short-term memory deficits in coding, storing and retrieving these representations (Gathercole \& Baddeley, 2014).

According to recent studies, dyslexic and normal readers differ in terms of processing visual and auditory information, as the large cell visual pathway of dyslexics, presents some form of abnormality or dysfunction (Stein, 2001). Livingstone and Galaburda (1993), concluded that the large cells of the central nervous system of people with dyslexia were smaller in size and disorganized compared to normal people, a finding which was later confirmed in an in-vivo study by Giraldo-Chica et al., (2015). This hypothesis of magnocellular processing deficits has offered an alternative explanation of auditory and visual processing deficits. More specifically, the instability in focusing along with the problematic eye movement or increased density of visual stimuli result in visual deficits, which may also lead to 
auditory and motor problems (Ramus et al., 2003) as they carry information about motion, overall shape, and small light-dark changes.

In several studies over the last 20 years, electroencephalograph (EEG) has been used to study the physiology of the brain in patients with dyslexia, as it is non-invasive, painless, cheap and potentially wearable to record electrical activity of human brain from the scalp surface (Xing, Mccardle, \& Xie, 2012). Most studies focusing on EEG rhythms' changes have employed tasks associated with reading difficulties. For example, Rippon and Brunswick (2000) found that children with dyslexia showed increased frontal activity in a phonological task, and no differences were observed between the dyslexic group and the control group in a visual task. In addition, there was a marked increase in $\beta$ rhythm activity in the right parietal-occipital region in children with dyslexia when performing phonological in relation to the visual task (Papagiannopoulou \& Lagopoulos, 2016). The coherence of $\beta$ and $\gamma$ rhythms has been linked to more complex linguistic sub-processes, such as syntax or semantics. EEG findings from the literature study show increased (left) frontal and right temporal slow activity in the $\delta$ and $\theta$ bands and increased $\beta$ in F7 (Kandel et al., 2017).

A number of methods have been proposed in order to measure EEG signals in several populations through brain computer interface (BCI). One such device is the lightweight Emotiv EPOC+ wireless EEG system which has received the most empirical attention in a spectrum of different fields (Badcock et al., 2015). Concerning the exploration of the relationship between several forms of learning difficulties and EEG abnormalities there have been just a handful of researches using the Emotiv EPOC+. For example, Eroglu et al., (2018), found that the dyslexic group showed significantly lower complexity at the lowest temporal scale and at the medium temporal scales than did the control group.

\section{Objectives}

The purpose of this study is to investigate the brain function of young adults with dyslexia through innovative and non-invasive methods of functional imaging of the brain in phonological and morphological awareness tests, such as audiovisual discrimination tasks in a word-level.

\section{Methods}

\subsection{Data acquisition}

In this study, 26 right-handed young adults (mean average $21.32 \mathrm{y} / \mathrm{o}$ ) participated in this experiment, forming the Dyslexic group (12 students) and the Control group (14 students). All the subjects with dyslexia had undergone intervention at young age without reporting any dyslexia-related comorbidities. There were no major age or education-level differences, since all of them were university students in the School of Health Sciences. Written consent forms to participate in this study were obtained from all the subjects who participated on a voluntary basis. The recording was terminated as soon as a participant felt any discomfort with the device or the procedure. Each experimental session lasted 22 minutes on average depending on the time required by the participants to answer each question.

\subsection{EEG acquisition}

For the EEG recordings, the Emotiv EPOC+ head-set was used, a wireless neuro-signal acquisition device with 14 wet sensors ( +2 reference), capable of detecting brainwaves at $128 \mathrm{~Hz}$ sequential sampling rate. The participants were seated in a comfortable chair in front of a computer and a specialized technician set up the device following the instructions provided by the EmotivPRO Software, checking regularly the quality of the connectivity in the beginning and during the recording. The felt pads were placed in the scalp according to the International 10-20 System (AF3, F3, F7, FC5, T7, P7, O1, AF4, F4, F8, FC6, T8, P8 and O2), using saline liquid solution on all felt pads of each sensor (Figure 1). However, due to loss of connectivity the F8 electrode was isolated and rejected and so was the corresponding channel, F7 to maintain the symmetry of the recording.

Figure 1. Regions of Interest according to the electrode sites. (Blue: Left frontal, Orange: Left temporal, Red: Left occipital, Green: Right frontal, Purple: Right temporal, Yellow: Right occipital, Grey: Rejected channels).

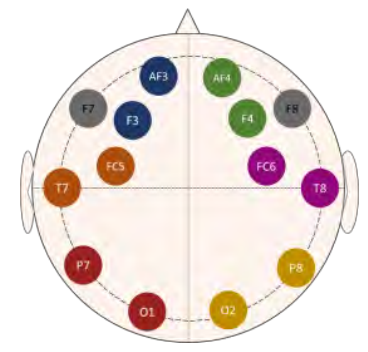


The recordings were made with the montage, according to the connected mastoids, and the EEG signals were saved in ".edf" format. A Butterworth notch filter is applied to remove $50 \mathrm{~Hz}$ power line noise oscillations from EEG signals and a $0.5 \mathrm{~Hz}$ high-pass digital FIR filter to remove low frequency oscillations. Next, five FIR filters of similar design are designed to allow frequencies within a certain range and attenuate frequencies outside that range. The five bandwidth filters $(\delta \approx 0.5-4 \mathrm{~Hz}, \theta \approx 4-8$ $\mathrm{Hz}, \alpha \approx 8-12 \mathrm{~Hz}, \beta \approx 13-30 \mathrm{~Hz}$, and $\gamma \approx 30-60 \mathrm{~Hz}$ ) are designed in relation to the $5 \mathrm{EEG}$ rhythms, trying to export spectral characteristics to each subband of frequencies to be investigated.

\subsection{Material}

The material included in the software consists of 60 triads of words, which have been selected meeting strict phonological, morphological and semantic criteria taking into account the difficulties encountered by people with dyslexia (Asvestopoulou et al., 2019). More specifically, the given words

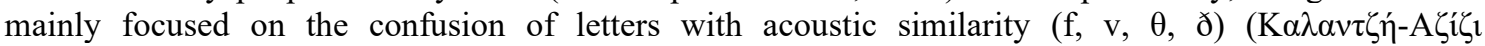

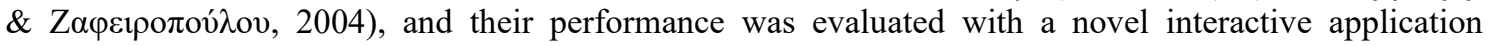
measuring audiovisual discrimination of words in two experimental conditions.

\subsection{Auditory discrimination task}

In the first experimental auditory discrimination task, participants were asked to differentiate verbally-presented words containing phonemes with common phonological characteristics (eg fo'vame, fo' $\partial a m e$, fo' $\theta$ ame). Participants saw 3 boxes with written numbers in a row on a computer screen, asking them to choose the number that corresponded to the word they thought was correct in predefined time limit of 10 seconds. The verbal instruction given was the following: "choose the right word you hear".

\subsection{Visual recognition task}

In the second experimental task, which assessed visual recognition, the participants saw 3 words in a row on the screen, and had to implicitly read them as carefully as possible choosing the one that they thought had the correct spelling in predefined time limit ranging from 5 to 10 seconds. The on-screen instruction was: "Choose the right word you see".

\section{Results}

Aiming to examine the differences in EEG recordings between the two sets of groups in (i) auditory discrimination and (ii) visual recognition of words, we performed the t-test as the data were normally distributed.

(i) Regarding the auditory discrimination task we observed statistically significant differences in both hemispheres. More specifically, in the left hemisphere differences were found in the left temporal lobe in $\beta(p=.005), \gamma(p=.002)$ and $\delta(p=.017)$ rhythms, in the left occipital lobe in $\beta(p=.02)$, rhythm, and in the right prefrontal area in $\alpha(p=.02), \beta(p=.05)$ and $\gamma(p=.04)$ rhythms, respectively. Students with dyslexia reported higher mean scores only in $\delta$ rhythm in the left temporal lobe, and in $\alpha, \beta$ and $\gamma$ rhythms in the right prefrontal area of the hemisphere (Table 1).

Table 1. T-test examining the correlation of brain regions and rhythms between Control and Dyslexic groups in Auditory discrimination task.

\begin{tabular}{|c|c|c|c|c|c|c|}
\hline & \multicolumn{2}{|c|}{ Control $(n=14)$} & \multicolumn{2}{|c|}{ Dyslexic $(n=12)$} & \multicolumn{2}{|c|}{ df 25} \\
\hline & \multicolumn{6}{|c|}{ Left Hemisphere } \\
\hline & $\mathrm{M}$ & $\mathrm{Sd}$ & $\mathrm{M}$ & $\mathrm{Sd}$ & $\mathrm{t}$ & Sig \\
\hline T7_ $\delta$ & 0.556 & 0.234 & 0.771 & 0.100 & -2.585 & .017 \\
\hline T7__ $\beta$ & 0.113 & 0.082 & 0.032 & 0.014 & 2.890 & .005 \\
\hline T7_ $\gamma$ & 0.133 & 0.093 & 0.031 & 0.015 & 3.255 & .002 \\
\hline \multirow{2}{*}{ O1_ $\beta$} & 0.091 & 0.031 & 0.054 & 0.027 & 2.487 & .027 \\
\hline & \multicolumn{6}{|c|}{ Right Hemisphere } \\
\hline AF4_ $\alpha$ & 0.014 & 0.011 & 0.029 & 0.014 & -2.569 & .020 \\
\hline AF4_ $\beta$ & 0.020 & 0.014 & 0.052 & 0.024 & -3.758 & .005 \\
\hline $\mathrm{AF} 4 \_\gamma$ & 0.026 & 0.088 & 0.047 & 0.114 & 2.223 & .040 \\
\hline
\end{tabular}

(ii) Concerning the visual task, statistically significant differences were evident in the left temporal lobe in $\beta(p=.02), \gamma(p=.04)$ rhythms, in the occipital lobe in $\alpha(p=.01), \beta(p=.01)$ and $\delta(p=.02)$ rhythms, in the left parietal lobe in $\beta(p=.02)$ rhythm, and in the right occipital lobe in $\delta(p=.01), \beta(p=.01)$ and $\gamma(p=.03)$ rhythms. The students with dyslexia reported higher mean scores only in the $\delta$ rhythm of both the left and right occipital lobe (Table 2). 
Table 2. T-test examining the correlation of brain regions and rhythms between Control and Dyslexic groups in Visual recognition task.

\begin{tabular}{|c|c|c|c|c|c|c|}
\hline & \multicolumn{2}{|c|}{ Control $(n=14)$} & \multicolumn{2}{|c|}{ Dyslexic (n=12) } & \multicolumn{2}{|c|}{ df 25} \\
\hline & \multicolumn{6}{|c|}{ Left Hemisphere } \\
\hline & M & $\mathrm{Sd}$ & M & $\mathrm{Sd}$ & $\mathrm{t}$ & Sig \\
\hline T7_ $\beta$ & 0.142 & 0.088 & 0.064 & 0.060 & 2.334 & .020 \\
\hline T7_ $\gamma$ & 0.170 & 0.111 & 0.080 & 0.081 & 2.090 & .040 \\
\hline O1_ $\delta$ & 0.420 & 0.132 & 0.595 & 0.206 & -2.499 & .021 \\
\hline O1_a & 0.045 & 0.018 & 0.034 & 0.011 & 2.567 & .011 \\
\hline O1_ $\beta$ & 0.106 & 0.025 & 0.068 & 0.039 & 2.813 & .010 \\
\hline \multirow[t]{2}{*}{$\mathrm{P} 7 \_\beta$} & 0.140 & 0.076 & 0.079 & 0.048 & 2.121 & .032 \\
\hline & \multicolumn{6}{|c|}{ Right Hemisphere } \\
\hline $\mathrm{O} 2 \_\delta$ & 0.355 & 0.152 & 0.538 & 0.205 & -2.612 & .015 \\
\hline $\mathrm{O} 2 \_\beta$ & 0.123 & 0.041 & 0.028 & 0.019 & 3.136 & .011 \\
\hline $\mathrm{O} 2 \_\gamma$ & 0.131 & 0.106 & 0.090 & 0.062 & 2.280 & .033 \\
\hline
\end{tabular}

\section{Discussion}

The aim of this study was to track brain activity in the regions of interest, testing differences in brain rhythms as they were recorded through a Brain Computer Interface device, between young adults with dyslexia and a control group across two experimental conditions (auditory discrimination and visual recognition).

The results showed statistically significant differences in brain regions in both hemispheres between the two groups, revealing the heterogeneity of rhythms' activation in different regions (Perrachione et al., 2016). The findings in both experimental conditions are in line with previous researches reporting a left occipito-temporal hypoactivation converging on the same brain regions associated with the reading deficit (Paulesu et al., 2014; Zakopoulou et al., 2019).

What is evolutionary in this study, is the attempt to further investigate the rhythms' activation within these brain regions, where students with dyslexia exhibited lower $\beta$ and $\gamma$ rhythms in the left occipito-temporal lobes, during both auditory and visual tasks, suggesting that these rhythms are linked with difficulties in the phonological and reading processes as well as with failure of left posterior brain systems (Shaywitz et al., 2002). Similarly, a reduced $\alpha$ activity in the left occipital region was found only during the visual task, indicating a relationship between $\alpha$ rhythm, cognitive performance (Riviello et al., 2011), and brain maturation (Pineda, 2005).

Interestingly, during the auditory task an increased $\delta$ rhythm was found in the left temporal region, while similarly high $\delta$ rhythm was found in the left occipital region during the visual task. Being in line with Gori's (Gori et al., 2015) and Kandel's (Kandel et al., 2017) results, these findings enrich the assumption that the left occipito-temporal slow activity reveal a strong interaction between auditory processing difficulties and reading impairments.

\section{Conclusion}

$\alpha, \beta$ and $\delta$ EEG bands were used to define unique brain activations and related possible phonological and reading impairments, in adults with dyslexia, during auditory and visual tasks. Such evidence would be relevant for the theory of magnocellular processing, which postulates the coexistence of auditory and visual processing deficits as indicative of a broadly distributed dysfunction in the "neural signature" of dyslexia.

\section{References}

American Psychiatric Association, DSM-5 Task Force. (2013). Diagnostic and statistical manual of mental disorders: DSM-5 TM (5th ed.). American Psychiatric Publishing, Inc.

Asvestopoulou, T., Manousaki, V., Psistakis, A., Smyrnakis, I., Andreadakis, V., Aslanides, I. M., \& Papadopouli, M. (2019). Dyslexml: Screening tool for dyslexia using machine learning. arXiv preprint arXiv:1903.06274. 
Badcock, N. A., Preece, K. A., de Wit, B., Glenn, K., Fieder, N., Thie, J., \& McArthur, G. (2015). Validation of the Emotiv EPOC EEG system for research quality auditory event-related potentials in children. Peer Journal, 3, e907.

Eroğlu, G., Aydın, S., Çetin, M., \& Balcisoy, S. (2018). Improving cognitive functions of dyslexics using multi-sensory learning and EEG neurofeedback. In 2018 26th Signal Processing and Communications Applications Conference (SIU) (pp. 1-4). IEEE.

Gathercole, S. E., \& Baddeley, A. D. (2014). Working Memory and Language. Psychology Press.

Giraldo-Chica, M., Hegarty, J., \& Schneider, K. (2015). Morphological differences in the lateral geniculate nucleus associated with dyslexia. NeuroImage: Clinical, 830-836.

Gori, S., Seitz, A., Ronconi, L., Franceschini, S., \& Facoetti, A. (2015). The causal link between magnocellular-dorsal pathway functioning and dyslexia. Journal of Vision, 15(12), 195-195.

Kandel, S., Lassus-Sangosse, D., Grosjacques, G., \& Perret, C. (2017). The impact of developmental dyslexia and dysgraphia on movement production during word writing. Cognitive Neuropsychology, 34(3-4), 219-251.

Livingstone, M., \& Galaburda, A., (1993). Evidence for a magnocellular defect in developmental dyslexia. Annals of the New York Academy of Sciences, 682, 70-82.

Papagiannopoulou, E. A., \& Lagopoulos, J. (2016). Resting State EEG Hemispheric Power Asymmetry in Children with Dyslexia. Frontiers in pediatrics, 4, 11.

Paulesu, E., Danelli, L., \& Berlingeri, M. (2014). Reading the dyslexic brain: multiple dysfunctional routes revealed by a new meta-analysis of PET and fMRI activation studies. Frontiers in Human Neuroscience, 8, 830 .

Paulesu, E., Démonet, J. F., Fazio, F., McCrory, E., Chanoine, V., Brunswick, N., Cappa, S. F., Cossu, G., Habib, M., Frith, C. D., \& Frith, U. (2001). Dyslexia: cultural diversity and biological unity. Science (New York, N.Y.), 291(5511), 2165-2167.

Perera, H., Shiratuddin, M. F., \& Wong, K. W. (2018). Review of EEG-based pattern classification frameworks for dyslexia. Brain informatics, 5(2), 4.

Perrachione, T. K., Del Tufo, S. N., Winter, R., Murtagh, J., Cyr, A., Chang, P., ... \& Gabrieli, J. D. (2016). Dysfunction of rapid neural adaptation in dyslexia. Neuron, 92(6), 1383-1397.

Pineda, J. A. (2005). The functional significance of mu rhythms: translating "seeing" and "hearing" into "doing". Brain research reviews, 50(1), 57-68.

Ramus, F., Rosen, S., Dakin, S. C., Day, B. L., Castellote, J. M., White, S., \& Frith, U. (2003). Theories of developmental dyslexia: insights from a multiple case study of dyslexic adults. Brain, 126(4), 841-865.

Rippon, G., \& Brunswick, N. (2000). Trait and state EEG indices of information processing in developmental dyslexia. International journal of psychophysiology: official journal of the International Organization of Psychophysiology, 36(3), 251-265.

Riviello, J. J., Nordli, D. R., \& Niedermeyer, E. (2011). Normal, EEG., and sleep: infants to adolescents, in Electroencephalography: Basic Principles, Clinical Applications and Related Fields, ed E. Niedermeyer Philadelphia, PA: Lippincott; Williams and Wilkins, 163-182.

Shaywitz, B. A., Shaywitz, S. E., Pugh, K. R., Mencl, W. E., Fulbright, R. K., Skudlarski, P., ... \& Gore, J. C. (2002). Disruption of posterior brain systems for reading in children with developmental dyslexia. Biological psychiatry, 52(2), 101-110.

Snowling, M. J. (2000). Dyslexia (2nd ed.). Blackwell Publishing.

Stein, J. (2001). The magnocellular theory of developmental dyslexia. Dyslexia, 7(1), 12-36.

Xing, S., McCardle, R., \& Xie, S. (2014). The development of EEG-based brain computer interfaces: potential and challenges. International journal of computer applications in technology, 50(1-2), 84-98.

Zakopoulou, V., Vlaikou, A. M., Darsinou, M., Papadopoulou, Z., Theodoridou, D., Papageorgiou, K., ... \& Michaelidis, T. M. (2019). Linking Early Life Hypothalamic-Pituitary-Adrenal Axis Functioning, Brain Asymmetries, and Personality Traits in Dyslexia: An Informative Case Study. Frontiers in human neuroscience, 13, 327.

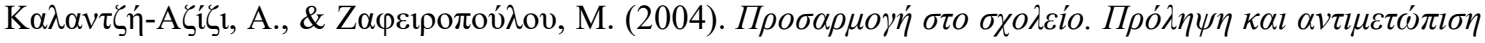

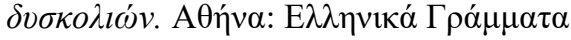

